\title{
Reproducción e imagen de la mujer. Crítica de género en demografía
}

\section{Graciela Sarrible}

U niversitat de Barcelona. Facultat de Ciències Polítiques. D epartament de Sociologia.

Diagonal, 690. 08034 Barcelona. Spain

\section{Resumen}

El artículo trata la cuestión del género en conexión con variables demográficas y las nuevas propuestas sobre el estatus y la posición de la mujer que afectan los niveles de fecundidad tanto en sociedades en desarrollo como en sociedades avanzadas.

Palabras clave: mujer, género, fecundidad, patriarcado.

\begin{abstract}
Reproduction and image of the woman. Gender's criticism in demography
The article deals with the question of gender in connection with demographic variables. N ew approaches to women status and social position might explain the fecundity level in advanced as well as non developed societies.
\end{abstract}

Key words: woman, gender, fertility, patriarchy.

\section{Sumario}

1. Respecto de la nupcialidad: mujeres devaluadas con hijos

2. La mujer reproductora: ¿el centro de la decisión?
3. La mujer activa: ¿antítesis de reproductora?

4. Los hombres: desigualdades por género

Bibliografía

En esta última década, las investigaciones en torno a la mujer y el planteamiento de la cuestión del género han prestado por fin atención a las cuestiones demográficas. En general, estos trabajos giran al rededor de la nueva propuesta sobre el estatus y la posición de la mujer como variables explicativas de los cambios y las alteraciones de cuestiones demográficas, sobre todo la fecundidad (Federici y otros, 1985). 
Los estudios insisten en la incorporación de una variable - el estatus de la mujer - que hasta el momento no había sido tenida en cuenta en las explicaciones de cuestiones pendientes. Los cambios en los niveles de la fecundidad, tanto en las sociedades en desarrollo como en las sociedades avanzadas no han sido explicados todavía satisfactoriamente por las distintas propuestas teóricas existentes. Las opciones provenientes de las escuelas económicas han recibido mayor atención. Esto se debe a la proyección de estas escuelas más que a la capacidad explicativa de estas opciones para las cuestiones privadas y familiares.

El estatus de la mujer no ha tenido suficiente eco en las opciones políticas. A pesar de que la propuesta es clara: a mayor igualdad, niveles de fecundidad más próximos al reemplazo; los gobiernos no han considerado esta relación al delinear sus políticas de población. Al contrario, prefieren considerar variables anacrónicas con resultados de escasa eficacia. Este enunciado no ha sido refutado hasta el momento, pero más que una teoría, constituye un factor explicativo complementario, entre tantos otros, que no había sido considerado y que pone de relieve - mejor que otros lo han hecho- las diferencias entre las distintas sociedades, incluso en el contexto europeo.

La hipótesis básica de estas formulaciones considera que cuando el estatus de la mujer es más igualitario respecto del hombre, los niveles de fecundidad podrían tender a una cierta estabilidad en torno al reemplazo de las generaciones. Si la fecundidad es elevada, el patriarcalismo imperante obligaría a las mujeres a tener más hijos de los deseados. Si la fecundidad es muy baja, es que la mujer no tiene suficientes garantías, ayudas o colaboración ni por parte del Estado ni por parte de su pareja.

Si la fecundidad se sitúa en torno al mítico 2,1 hijos por mujer - como descendencia final al canzada - podría vincularse con una situación que sin ser la ideal sí es la más adecuada para que la mujer trabaje y sea también madre, con los menores inconvenientes posibles. Los países escandinavos, que han sido tomados como ejemplo en múltiples ocasiones, constituyen la demostración pal pable de que la maternidad resulta compatible con altos niveles de participación de la mujer en la población activa, en sociedades avanzadas.

Si el patriarcalismo obliga a las mujeres a reproducir la sociedad más allá de los propios deseos de la población femenina, no va a responderse en este artículo - por ser una polémica que excedería este marco de reflexión- . Baste decir que es uno de los argumentos que se barajan políticamente en organizaciones internacionales, como explicación de la insuficiencia de resultados en el control de la fecundidad. Todos podemos estar intrínsecamente de acuerdo en que las mayores ventajas que otorguen la igualdad a la mujer en esas sociedades será positiva, independientemente de las consecuencias en el logro de los objetivos previstos respecto al crecimiento de la población de esas sociedades.

Esta explicación resulta crucial en el panorama europeo al enfrentar la situación del sur con la de los países escandinavos. Si el norte había tenido un descenso de la fecundidad más temprano, el sur lo ha tenido tarde y concentrado; de tal manera que la cuantía de los indicadores transversal es de fecundidad se considera relativamente reducida respecto de lo esperado. En estas condicio- 
nes y cuando algunos gobiernos desean - o quizás desearían- actuar para modificar estas tendencias, la pregunta obvia es cuál sería la política más efectiva. $\mathrm{H}$ asta ahora no existe evidencia en contra de que la mayor igualdad entre hombres y mujeres no constituya la mejor opción política en materia de fecundidad, sin menoscabo de las ventajas intrínsecas que esta elección conlleva. Si los gobiernos tomaran en cuenta esta propuesta, la igualdad recibiría un nuevo e insólito impulso.

Esta es una cuestión que, por su complejidad, haría falta considerar con bastante cautela. Lo que resulta anacrónico es que en este contexto donde la mejor situación de la mujer podría propiciar la recuperación de la fecundidad a nivel es que ciertas instituciones desearían, la mujer, como objeto de estudio y reflexión, sea tan mal tratada en los textos que se refieren a este tema. $D$ esde la nupcialidad, a la mortalidad, cuando la mujer presenta ventajas respecto al hombre, rara vez son vistas o interpretadas como tales. Por ejemplo, respecto a la mortalidad diferencial el hombre es presentado como más audaz y por eso se supone que fallece más. Argumentos insostenibles se siguen mencionando sin el más mínimo asomo de rubor.

En la cuestión de la fecundidad, en la que se basa la reproducción, el tratamiento es más despectivo o culpabilizador. Si la mayor participación de la mujer en la población activa tuviera como consecuencia la reducción de su descendencia, ¿se supone entonces que habría que retirarla del mercado de trabajo para que tuviera más hijos? La primera fue una interpretación de la evolución de las sociedades en términos de larga duración; pero la segunda propuesta no es defendible en ningún caso. La cantidad de investigaciones que sostienen lo contrario o muestran las fisuras de esta opción en la actualidad son innumerables.

Resulta paradójico que en esta situación, la imagen de la mujer que dan los estudios sea la de un ser culpabilizado por no tener exactamente los hijos que de ella se pretende. Toda la reproducción social pasa por una decisión errada de la mujer y hay que hacer al go para corregirla. El objetivo de este artículo es analizar y exponer la imagen tergiversada que se da de la mujer en cuestiones de reproducción. Esa imagen que surge de las teorías y de los trabajos de investigación es utilizada para intentar justificar ciertas propuestas políticas transmitidas, normalmente, a través de los medios de comunicación. Esa imagen no es más que la construcción social que determinada producción científica, lamentablemente la más difundida, hace de la mujer.

$\mathrm{N}$ o dejo de reconocer que se están publicando cada vez más trabajos con interpretaciones alternativas. Pero no siempre se critica lo establecido como una visión incorrecta de la imagen de la mujer, sino que se parte de estos supuestos como premisas para avanzar. Ello implica que no hay cuestionamientos de la base teórica de donde surge esa imagen distorsionada. Sin pretensiones de exhaustividad, este artículo pretende desconstruir esa imagen de la mujer que considera no sólo incorrecta, sino también no ajustada a la realidad. N o se trata pues de una análisis de la real idad, sino de la interpretación que se hace de esa realidad. Es un análisis de la construcción científica de esa categoría y de su imagen social. 


\section{Respecto de la nupcialidad: mujeres devaluadas con hijos}

La idea de mercado, aplicada a la elección y constitución de las parejas, resulta cuanto menos discutible. El hecho de que todos los solteros estén ahí para casarse también puede ser discutible. Respecto de la primera, es una interpretación que debemos a la teoría económica. N o pretendo contestar ideas que han sido premiadas con el $\mathrm{N}$ obel, pero sí la desvalorización que se atribuye a la mujer en determinadas condiciones.

Al considerar que todos, hombres y mujeres, están en un mercado para elegirse y casarse ello debería constituir un punto de partida similar para cualquiera de las dos subpoblaciones (Weiss, 1994). Sin embargo, si la primera vez que uno deja de ser soltero las oportunidades aparentemente están bastante equilibradas (salvo al teraciones históricas o sociales específicas como períodos de guerra o fuertes migraciones diferenciales por sexo), cuando se trata de reincidir en el matrimonio, las valoraciones de cada género se distancian considerablemente porque las frecuencias con que optan por un segundo matrimonio también los diferencian.

Es cierto que la mayoría de los jóvenes sigue casándose en España (aunque puede que ya no sea verdad dentro de al gún tiempo). También es factible que las jóvenes generaciones se casarán seguramente menos que sus amigos de más edad, entre otras razones porque podrán optar por variadas formas de convivencia 0 de hogares. En la primonupcialidad (o nupcialidad de las personas solteras) no se manifiestan, entonces, agravios comparativos en el tratamiento de cada uno de los géneros.

Cuando se trata de segundas parejas, dado que no todos los que podrían reincidir lo hacen, la frecuencia de cada sexo varía considerablemente. LoS segundos matrimonios están aumentando, en general en Europa, en términos absolutos y descendiendo en términos relativos. Ello quiere decir que si bien cada vez hay más gente divorciada (ya que no se trata de ningún incremento de la viudez temprana), las personas con este estado civil no tienden a volver a casarse en la misma proporción que lo hacían sus homónimos hace algunos años. Las diferencias entre hombres y mujeres resultan significativas. El hombre se casa más frecuentemente que la mujer, en segundas nupcias, antes de los cincuenta años.

La pregunta, entonces, es si todos quieren casarse por igual y el hombre lo concreta más que la mujer o si, a la inversa, los deseos de contraer nuevas nupcias son distintos por género. $\mathrm{H}$ asta ahora, se presuponía que el hombre se casaba más porque era más valioso. Se afirmaba que una mujer divorciada (sobre todo si tenía hijos y estaban a su cargo) se devaluaba considerablemente en ese mercado y si no se casaba es porque no encontraba quien la quisiera.

En realidad, esta interpretación parte de la premisa que la segunda vez la gente se comporta igual que la primera y por lo tanto cabría aplicarle el mismo método de probabilidades y de estudio de frecuencias. Ahora bien, estimo que los deseos de casarse pueden ser distintos por género, cuando no se trata de la primera vez. En términos de opción racional, es lógico que el hombre quiera 
casarse porque es el estado civil con menor mortalidad y por lo tanto tiene protección garantizada. En la mujer, en cambio, no se aprecian ventajas en este sentido en lo que respecta a la disminución de la probabilidad de morir por el hecho de estar casada. Q uizás en lo económico, la vida en pareja constituya una ventaja para ambos.

Existen otros factores de riesgo (según las propuestas económicas tan a la moda) que podrían empujar a los hombres 0 a las mujeres a contraer nuevas nupcias. El error es considerar que los factores que benefician a unos (los hombres) también lo hacen con las mujeres. Suponer que las prioridades y los valores son idénticos y que por lo tanto las opciones que eligen también deberían serlo, es una premisa cuanto menos cuestionable. Si el punto de partida es dife rente, resulta injusto juzgar ambos casos con los mismos parámetros y presumir una igualdad inicial que no existe.

Si hasta ahora se casaba - por primera vez- el $90 \%$ de los efectivos de las generaciones - proporción observada a los cincuenta años- , considerar que existía un impulso o deseo de seguir el comportamiento mayoritario puede que fuera adecuado. Q uizás la revisión debería hacerse respecto de los que no se casaban. Si la mayoría se casa debe ser porque así lo desea. Pero, los que no se casan puede que también así lo deseen. A esta altura de la preeminencia del individualismo resulta difícil considerar que la gente que no se casa es porque tiene defectos (está devaluada en el mercado). Por suerte, se ha avanzado lo suficiente como para reconocer que existen otras opciones en la vida de las personas que estar casadas.

Entonces, ¿por qué esta idea de no obligatoriedad no persiste en los segundos casamientos? ¿Por qué las mujeres no pueden reaccionar de manera diferente frente a una ruptura? D e hecho, lo hacen. Si uno de cada cuatro hombres que se ha casado anteriormente elige la cohabitación como segunda opción, entre las mujeres la proporción se el eva a casi una de cada tres (Sarrible, 1995). ¿Es que la cohabitación vale menos que el matrimonio, o es que no se puede detectar de la misma manera porque no se registra? Q ue las mujeres opten menos por un segundo matrimonio no significa que no vivan en pareja, sino que pueden elegir otra clase de relación con o sin institucionalización o convivencia.

Sin embargo, la principal diferencia entre hombres y mujeres después de un divorcio es la tenencia de los hijos - cuando ha habido descendencia- . En la mayoría de los casos, los hijos permanecen con la madre y el padre pasa a vivir, generalmente, en un hogar unipersonal, por poco tiempo. Viejas interpretaciones querían ver la permanencia de esos hijos con la madre como el principal factor de desvalorización de la mujer ${ }^{1}$.

Q ue los hijos vivan con la madre significa tiempo y dedicación, mientras que el hombre dispone de todas sus posi bilidades para buscar nuevas parejas. Pero ello no significa que la mujer valga menos en un mercado de intercambios, sino

1. De los matrimonios disueltos por separación en España, el $73 \%$ tenía hijos menores y en más de ocho de cada diez casos permanecieron con la madre (Encuesta Socio-demográ fica, 1991). 
que puede tener otras prioridades. M ientras que los hombres divorciados constituyen hogares unipersonales en un tiempo intermedio entre el divorcio y la nueva pareja, la mujer lo hace después de la partida de los hijos y por lo tanto a una edad más tardía. El hecho de vivir solos cambia según el estado civil de cada sexo (H askey, 1990).

La mujer merece el mismo respeto que el hombre por sus decisiones. Las investigaciones han puesto en evidencia que las condiciones de vida de mujeres y hombres no son las mismas. Las desigualdades sociales influyen en la situación de la mujer. Sus valores o prioridades resultan diferentes. En estas circunstancias sería extraño que tomaran las mismas decisiones. El hecho de no proceder como el hombre no puede implicar, en ningún caso, que la mujer valga menos. Si sus condiciones no son igualitarias, las opciones que haga estarán en función de todo su entorno y no pueden ser idénticas a las del hombre.

\section{La mujer reproductora: iel centro de la decisión?}

Si parece que la mujer no tiene ventajas por tener hijos, tampoco las tiene por no tenerlos. La mujer que está casada y no es madre es requerida constantemente, sobre todo en el más próximo entorno de la familia, por una decisión que provoca desde rechazo hasta consternación. Como es la mujer la que se queda embarazada, al hombre no se le cuestiona en la misma medida.

Evidentemente, no tener hijos - sobre todo cuando se está casada - es una decisión meditada. Si todavía no hay respuesta a la cuestión del porqué se tienen hijos, estamos más cerca de saber porqué las personas no los tienen. D ecidir a contracorriente de la sociedad implica una reflexión profunda, una racionalización de los proyectos de vida que normalmente se atribuye a la decisión de constituir una descendencia. M ás difícil que constituirla parece ser el hecho de negarse a tenerla (Sarrible, 1995).

La mujer parece ser el centro de las decisiones de reproducción (y es muy factible que así sea). Ello significa adaptarse a unos cánones o model os predeterminados por ciertas instituciones (gobiernos u organismos internacionales). En los países como España e I talia que han registrado una relativamente reducida fecundidad transversal en la última década - no alcanzaría en el largo plazo para mantener el número de efectivos de la población, si esta fuera cerra da- , los gobiernos no han desarrollado políticas concretas para intentar alterar e invertir estas tendencias.

En los textos, la mujer aparece como culpable de tener muchos hijos. Se afirma que la fecundidad es elevada en los regímenes patriarcales. Pero además de patriarcales, esos regímenes tienen otras características, como fundamentalmente agrarios, industrialización reducida, escasa participación femenina en la población activa, escolarización femenina incompleta o nula, etc. El mejoramiento de la situación de la mujer se presenta como la panacea para reducir el ritmo de crecimiento (Salas, 1985).

Es cierto que la mujer no es independiente en esos países, pero tanto el hombre como la mujer están sujetos a condicionantes sociales que pueden 
influir en sus niveles de fecundidad. ¿Q ué quiere decir, entonces, que la mujer no decide? Primero, que se le impone el número de la descendencia y que lo hace el hombre. Segundo, que ella desea una cosa pero está sujeta y actúa según se le exige. Tercero, que si de ella dependiera haría otra cosa. ¿Por qué no pensar que los hombres si fueran libres de esos mismos condicionamientos que pesan sobre la mujer, también actuarían de otra manera?

Es difícil no culpabilizar al régimen patriarcal. Pero conceder una «libertad» desde arriba, para que la consecuencia sea reducir la tasa de crecimiento de la población de una determinada sociedad, puede no resultar tan efectivo como se pretende. Toda la sociedad debe cambiar, tanto las mujeres como los hombres.

Artículos sobre C hina, tanto científicos como declaraciones políticas, abundan en esta idea de que la fecundidad se reduciría notablemente si se dejara decidir a las mujeres (W olf y Ying-C hang, 1994). En el caso de este país, resulta, cuanto menos, paradójico. Se ha impuesto la política del hijo único como último recurso para desacelerar el crecimiento de la población. En el largo plazo y si esta política fuera realmente efectiva, significa el envejecimiento de la población al reducirse considerablemente los efectivos de las generaciones más jóvenes respecto de las anteriores.

¿Es que las mujeres en China, tienen la culpa del ritmo de crecimiento? ¿Y las preferencias de sexo en materia de fecundidad o los deseos de tener un hijo varón, no tienen nada que ver con esto? En varias sociedades asiáticas, estas preferencias implican la imposibilidad de reducir las probabilidades de agrandamiento de la descendencia cuando los anteriores nacimientos son de niñas².

El feminismo ha criticado al patriarcalismo, pero también lo había hecho H egels en el siglo pasado y la escuela de Frankfurt. ¿Por qué, entonces, en un área tan trillada no se ha avanzado más? D iría que por la misma razón por la que en ocasiones se impone la reducción del ritmo de crecimiento de la población. Se quiere controlar al Tercer M undo y se teme el poder que pudiera surgir de un número elevado de personas. D e la misma manera, se podría temer a las mujeres si decidieran ejercer su control sobre la esfera privada (convivencia en pareja y descendencia).

Los estudios de la mujer y el sistema de género se producen en un campo donde parece reclamarse sólo más justicia y equidad. Lo que no he visto es que se proponga que las mujeres tomen el control y decidan sobre sí mismas. El problema de la población no se plantea como un problema eminentemente femenino - la mujer controlará su propia fecundidad y la reproducción social- sino macrosocial, de gobiernos, de organismos; algo que está por encima de los individuos y necesita de la alta esfera política para definir una respuesta.

2. Las probabilidades de agrandamiento miden el riesgo de tener un hijo más. Por ejemplo, si se tiene uno, será el segundo. Pueden depender del sexo de los niños ya nacidos en los casos de fuerte y tradicional preferencia por uno de ellos. 
El feminismo siempre ha reivindicado el control del cuerpo de la mujer, pero lo ha hecho desde una perspectiva individual. No se han sumado las individualidades Ilegando a un agregado social. Se pide que cada mujer haga lo que desea. Pero no existe enfrentamiento notorio contra el poder, que impone políticas, metas y límites que se transforman en una forma de presión social contra las decisiones individuales y libres.

Así como la mujer es la reproductora, en términos biológicos, también parece que es la máxima responsable de la educación y la crianza de los hijos. Es cierto que las madres se quedan con los hijos después de la disolución del vínculo de pareja porque lo piden, pero ello no implica la falta de relación o de responsabilidad de los padres.

Los actuales movimientos de padres separados reivindican mayor participación en la crianza de los hijos, más tiempo y más dedicación (Sarrible y Guillen, 1995). Estas reivindicaciones justas, chocan contra una realidad de apatía y abandono. Los padres frecuentan cada vez menos a sus hijos, según pasa el tiempo desde que dejaron de convivir con ellos, según datos de otros países (Roussel, 1991).

C reo que es un supuesto bastante acertado considerar que los niños necesitan a ambos padres, a pesar de que no convivan con ellos. Sin embargo, parece como si el tiempo dedicado por la madre tuviera otra calidad. Si la madre dedica tiempo, también puede hacerlo el padre. Resulta, en cambio, problemático que la responsabilidad de cuidar a los hijos sea exclusivamente femenina, en último término. El tiempo dedicado es desigual, pero no se trata de tender a una equidad en este aspecto. Compartir las responsabilidades tiene mayor importancia. Si la ley reconoce una paternidad compartida, que en la realidad también lo sea y no sólo en el momento de reclamar derechos frente a un conflicto.

Cuando se estudian las etapas de la familia desde el punto de vista de los niños, se insiste en la biografía de la madre, más que en la del padre (Bumpass, 1984). La idea subyacente sería que el hijo tendría que soportar los avatares de una cierta inestabilidad en las parejas de la madre y que a él le toca sufrir las consecuencias de esos cambios. Poco se dice de las historias personales de los padres, que rehacen parejas más frecuentemente y en un período de tiempo más corto. A pesar de que se analice la frecuencia de visitas del padre o su ausencia, se piensa en la pareja de la madre como sustituto del padre. Se espera que la madre no sólo encuentre pareja sino que sustituya al padre, que por no convivir con sus hijos, va desapareciendo de sus vidas.

La primera reflexión, en estos casos, es en torno a la expresión del sentimiento paternal por parte de los padres biológicos que no viven con sus hijos. Resulta sorprendente que la ausencia de convivencia marque una etapa en que las frecuentaciones se van haciendo cada vez más escasas. Las responsabilidades no se diluyen como sucede con el tiempo dedicado a los hijos. La segunda cuestión que se plantea es que las parejas de la madre pueden reemplazar a los padres ausentes sin consecuencias psicológicas (M ott, 1990). Ello implica, 
entonces, que la madre tiene que encargarse además de serlo, de encontrar otro padre en caso de abandono del biológico.

Si la mujer parece la máxima responsable de los desequilibrios de pobla ción (al reproducirse más o menos de lo debido), la máxima responsable tanto de la maternidad como de la paternidad, todo ello no parece que se traduzca en un papel central o en una valoración positiva de su género, ni tampoco por ser muchas veces el eje de ciertos tipos de familia. Sin embargo, ello no impide que el hombre sea valorado por otras cualidades, sobre todo en sociedades donde la relación de los géneros está jerarquizada.

Los estudios de preferencia de sexo en materia de fecundidad obedecen a la lógica razón de que existen en ciertas culturas, sobre todo asiáticas, una imperiosa necesidad de tener descendencia de varones por razones tradicional es y religiosas (C oale y Banister, 1994). Esa escala jerárquica donde los sentimientos tradicionales llevan a distintas valoraciones de la descendencia por razones de sexo, no ha sido comprobada en 0 ccidente. No es excepcional, sin embargo, leer artículos de difusión donde se presume que de existir posibilidades de elegir el sexo del futuro embrión se elegirían hombres. Craso error. La Ley de Reproducción Asistida prohíbe esta selección, pero no explicita las razones del miedo ni el sentido del desequilibrio posible.

En la medida en que las encuestas de fecundidad se hacen a mujeres (y los varones no son interrogados), la preferencia de las mujeres por tener hijas mujeres en España no resulta sorprendente, en determinadas circunstancias. Según Ios datos de la Encuesta de Fecundidad de 1977, cuanto mayor es el número de hijos que ha tenido una mujer, más fuerte es el deseo de tener hijas ${ }^{3}$. Cuando, en 1985, se preguntó - en la subsiguiente Encuesta de Fecundidad- a las mujeres embarazadas qué preferencia de sexo tenían, casi la mitad no manifestó ninguna $(48,61 \%)$. Pero el resto no se distribuyó equitativamente, puesto que la preferencia por los varones fue del $18,85 \%$, mientras que por las mujeres alcanzó el 32,54\%.

Estas cuestiones pueden resultar superfluas en 0 ccidente en términos políticos, por varias razones. Primero, porque la fecundidad es tan reducida que la preferencia de sexo no parece incrementar el número de hijos, de acuerdo con el sexo de los nacimientos previstos. Segundo, porque por ley la selección es ilegal. Pero, en términos de imagen, que es lo que se analiza aquí, esta fal sa idea no resulta ni superflua ni intrascendente.

Q uizás, el problema es que los hombres no hayan sido encuestados. Si así se hiciera, las posibilidades básicas de combinación de las respuestas serían dos. Si están de acuerdo con la mitad de las mujeres que no opta ni por un sexo ni por el otro, entonces la idea de la preferencia de hijos varones en España sería totalmente falsa. Si, en caso contrario, prefirieran varones, tendríamos a la

3. Según los datos del cuadro 3.44, cuando se han tenido dos hijos y se desean tener más por razones de sexo, el 18,7\% desea un varón y el 36,9\% desea una mujer. Cuando se han tenido más de 3 , estas proporciones cambian: 6,5\% tendrían otro hijo porque desean un varón y 34,2\% en el caso de una mujer. 
población dividida por opiniones encontradas. Pero, en ningún caso, sería la uniformidad que se pretende en una identificación sin sentido con ciertas tradiciones de la lejana cultura oriental.

Esta manía de centrar todos los aspectos de la fecundidad en la mujer perjudica tanto a unos como a otros. A la mujer, porque aparece culpable de todo cuanto pasa. Pretenden que sea la única responsable y en esa medida encaminan y dirigen todas las políticas hacia ellas. En el caso de los hombres, su ausencia en ciertas políticas les perjudica, ya que deberían ir dirigidas al grupo familiar completo o deberían considerar ambos miembros de una pareja - cuando existe. Se comprende bien que si hay madres solteras y mujeres divorciadas, así como viudas jóvenes que encabezan familias monoparentales en 0 ccidente se les preste más que atención, apoyo. Pero si lo que se busca es actuar o influir en los niveles de fecundidad - cosa por sí bastante discutible- debería atenderse a la mayoría de las personas en edad fértil.

\section{La mujer activa: zantítesis de reproductora?}

La teoría de la transición demográfica sostiene que la incorporación de la mujer al mercado de trabajo conlleva el descenso de la fecundidad. D esde entonces ha pasado mucho tiempo y han cambiado muchas cosas. Lo que pudo ser cierto en la evolución de las sociedades europeas durante un período, puede no serlo más en la actualidad.

Esta relación todavía se plantea para los países en desarrollo, pero ahí tampoco está clara la relación, ni es directa ni lineal. Cuando la mujer trabaja en el campo, en tareas agrícolas lo hace igual o más que el hombre, pero tiende a menospreciar su trabajo y a no declararse activa en las encuestas. En estos países donde el sector primario recluta todavía abundante mano de obra, la estructura familiar tiene relación con los niveles de fecundidad.

Las familias campesinas - considerando cómo organizan la producciónno pueden tener el mismo trato - como objeto de estudio- que las familias urbanas que trabajan en otros sectores y conforman núcleos residenciales distintos (C aldwell, 1982). La ganancia de tener pocos o muchos hijos resulta completamente diferente en cada caso. Según Andorka (1978) - uno de los pocos análisis de la fecundidad a partir de la sociología- , lo que más influye es el costo diferencial de los hijos en cada caso.

Resulta evidente que no se puede generalizar ni extender la experiencia de unas sociedades europeas y occidentales a otras que están realizando todas las transiciones (industrial, social, demográfica) en un contexto geopolítico diferente. Las explicaciones que parten del desarrollo de 0 ccidente, 0 de las actuales sociedades avanzadas, no siempre encajan en esas otras sociedades. Tampoco las propuestas teóricas del pasado, que explicaron las primeras etapas de la transición resultan válidas hoy.

Con respecto a la estructura familiar, resulta importante, aún hoy, en los países occidentales, la ayuda que se pueda prestar las distintas generaciones. En el caso de las familias extensas, la corresidencia facilitaba la prestación de ser- 
vicios y la colaboración. En la actualidad, cuando prima la familia nuclear, la cercanía del domicilio con los abuelos influye en la frecuencia de los contactos y en las ayudas que mutuamente se puedan prestar (Logan y Spitze, 1994).

A pesar de que la evidencia indica que el nivel de participación de la mujer en la población activa ya tiene poco que ver con los niveles medios de fecundidad, sobre todo medidos transversalmente, los artículos más generales todavía se refieren a este aspecto de la teoría como válido y presentan los resultados contradictorios con ese enunciado como un hallazgo novedoso (K ravdal, 1994).

Las teorías económicas siguen imponiéndose en las explicaciones de la fecundidad aún cuando ciertos resultados las contradigan en algunos casos concretos (Wolf y Ying-C hang, 1994). La idea central es que el tiempo dedicado a la maternidad resulta incompatible con un empleo a tiempo completo. Ello implica o que no se tienen hijos o que se tienen pocos. La opción política que normalmente se menciona es la ayuda del Estado para mujeres que trabajan (Presser, 1989).

Q uizás este análisis resulte una simplificación excesiva de propuestas económicas mucho más complejas, pero la contradicción de ciertos resultados avalan una reelaboración de los enunciados. Si bien la idea del costo resulta central, las únicas relaciones que las investigaciones establecen con la actividad resultan ser siempre con la actividad femenina. El hombre debe de trabajar, por razones imperativas. Es la mujer la que aparentemente debería elegir y optar - entre el trabajo y la maternidad-. Los estudios de costos también se pueden plantear, en el mejor de los casos, como las horas «perdidas» por las mujeres al dedicárselas a sus hijos (J oshi, 1990).

$\mathrm{N}$ os encontramos permanentemente con la paradoja de que las cuestiones de la fecundidad y el empleo son exclusivamente femeninas. Las políticas, en este contexto teórico, deberían estar encaminadas a «ayudar» a las mujeres que trabajan fuera (Blau y Robins, 1989). D ependerá de la perspectiva que adopte cada opción política que la ayuda consista en que las mujeres sigan trabajando o dejen de hacerlo. Si la imagen subyacente de la mujer es que compite con el hombre por un puesto de trabajo, se provechará la maternidad para incentivar su retirada temprana del mercado de trabajo. Por el contrario, si se admite que maternidad y actividad femenina son más que compatibles, consustanciales en el mundo contemporáneo, entonces se intentará allanar los problemas para que ambas tareas puedan llevarse a cabo.

Evidentemente, los esfuerzos de los autores de los artículos se encaminan a prestar ayuda a la mujer y en implementar políticas que permitan mantener la descendencia en niveles de reemplazo o en retornar a ese nivel. En casos extremos y más dramáticos, se piensa en las condiciones de los hijos cuando el empobrecimiento de la familia está relacionado con su condición de monoparentalidad y el empleo de la madre resulta necesario a la supervivencia del grupo familiar (Eggebeen y Litcher, 1991). 


\section{Los hombres: desigualdades por género}

Cuando se tratan al gunas cuestiones sobre las mujeres, como la feminización de la pobreza, los hombres quedan al margen y parece que nada tuvieran que ver con estos procesos. La mayoría de las familias encabezadas por mujeres no provienen de madres solteras, sino de disoluciones de uniones donde la separación y el divorcio son cada vez más frecuentes frente a la viudez. Ello implica que el hombre, aunque no conviva con el grupo familiar, no es ajeno a la situación. A continuación se analizarán algunas cuestiones sobre las desigualdades entre los géneros.

En un artículo antológico, D avis y Van den O ever (1982) defendían al varón, perjudicado ante la mejor situación de supervivencia de la mujer. Cuando se trata la cuestión de la sobremoralidad masculina, las interpretaciones parecen extraídas de novelas románticas decimonónicas. La verdad es que no se ha encontrado explicación válida al hecho de que el sexo «débil» sea tan fuerte. Las propuestas se basaban en tipos de vida distintos para ambos sexos. A pesar de la creciente incorporación de la mujer a la población activa, ello no ha hecho descender sus expectativas de vida.

La cuestión de la sobremoralidad masculina, además, presenta otras desventajas. El hombre más protegido es el hombre casado, o sea, cuidado por una mujer. En cambio, no resultan tan evidentes las ventajas del matrimonio en términos de mortalidad diferencial para las mujeres. Los aspectos de sobre mortalidad por sexo y estado civil presentan las ventajas de ser mujer, pero las interpretaciones no perfilan, por ello, una mejor imagen femenina.

D avis, con toda razón, afirmaba que las diferencias biológicas entre los sexos no pueden ser completamente ignoradas cuando se reflexiona sobre la división del trabajo. Pensar en una situación utópica de igualdad entre los géneros no implica ignorar diferencias que persistirán. N o puede especularse sobre una situación idílica de igualdad social de los géneros, cuando la base biológica de las diferencias perdura: es siempre la mujer la que queda embarazada.

En el mismo sentido, U dry (1994) plantea hipotéticamente un intercambio de modelos de comportamiento atribuyendo a hombres los de mujeres y viceversa. L os investigadores de Estados U nidos, ya sean sociólogos o demógrafos, no menosprecian el sustrato biológico que existe, y que generalmente tiendea ser negado en otros casos. No todo es construcción social de los géneros. Las mujeres quedan embarazadas y los hombres no, y eso crea una diferenciación en el tiempo y dedicación al trabajo, al menos durante un período de su vida.

Q uizás el camino a la igualdad debería comenzar por reconocer las diferencias ineludibles que existen entre mujeres y hombres para poder construir otras opciones no tan utópicas - como las que sostienen una situación idéntica de partida que nunca podrá verificarse- . U dry sostiene que la naturaleza humana está condicionada por el género - se entiende que como construcción social- , sin menoscabo de los elementos biológicos.

$D$ avis fue más lejos una década antes al sostener que los nuevos roles sexuales se basan en los cambios demográficos que están afectando a cada uno de 
los sexos y pregonizó cambios en los modelos de nupcialidad para tender a un cierto equilibrio. Claro que al reconocer que la atracción sexual - considerada por él como elemento biológico- está vinculada con la elección de la pareja, deja abierta la posibilidad de que las elecciones respondan a patrones culturales y no a sus bienintencionadas propuestas.

En una cuantificación inusual, D avis destaca que el rol doméstico de la mujer perderá importancia al ser tan larga su vida y dedicar cada vez menos tiempo a la crianza de los hijos - que sólo ocupará un tercio de su vida adulta- . Esto implica repensar las prioridades y considerar la necesidad de un trabajo externo que debe llenar una parte importante de su tiempo de vida adulta.

Si se reflexionara en términos de cambios demográficos, tendencias y tiempo destinado a cada actividad y cada parte de la vida, sería más fácil construir alternativas basadas en la igualdad o si no, al menos en una distribución más equitativa de las tareas y de las obligaciones domésticas entre hombres y mujeres. Las ideas utópicas de igualdad futura no pueden ignorar las diferencias que permanecerán más allá de los cambios sociales y que implican que madres y padres son personas diferentes, deben estar encarnados por personas diferentes y no se trata de que uno asuma todos los papeles.

La necesidad de redefinir los géneros, en tanto construcción social, no es una formulación ni propia ni original por cuanto representa una reivindicación de una extensa literatura publicada. Lo que desearía señalar es que ese proceso debe estar acompañado por la construcción de categorías científicas donde la imagen de la mujer está deformada y devaluada. Esto no sólo es posible, sino realizable. No se trata de ir hacia una igualdad, sino hacia una diferenciación que no implique perjuicio para uno de los géneros.

\section{Bibliografía}

AN D ORKA, Rudolf (1978). D eteminants of Fertlity in Advanced Societies. Londres: M ethuen \& $C 0$.

BLAU, D avid M .; Ro BIN S, Philip K. (1989). «fertility, Employment and Child-Care Costs». D emography, vol. 26, núm. 2, p. 287-299.

BU MASS, Larry L. (1984). «C hildren and M arital D isruption: a Replication and U pdates. D emography, vol. 21, núm. 1, p. 71-82.

CADWELL, John C. (1982). Theory of Fertlity D edine. Londres: Academic Press.

C A ALE, Ansley; Banister, Judith (1994). «Five D ecades of M issing Females in China». Demography, vol. 31, núm. 3, p. 459-479.

D esal, Sonalde; Chase-Lan SD ale, P. Linsay; M IChael, Robert T. (1989). «M other or $M$ arket? Effets of $M$ aternal Employment on the Intelectual Ability of 4-yearold childrens». D emography, vol. 26, núm. 4, p. 545-561.

EgGEBeEn, David J.; LITHCER, D aniel T. (1991). «Race, Family Structure and Changing Poverty Among American Children». American Sociological Review, vol. 56, p. 801-817.

Federicl, Nora; CaselLI, Graziella; EgIDI, Viviana; Fong, Mónica (1985). «T he Status of Women, Population and D evelopment». N ewsetter, núm. 23-24, IU SSP, p. 77-98. 
H ASKEY, John C. (1990). «Les adultes vivant seuls: le cas de 30-59 ans en Grande Bretagne», capítulo 17. En La famille dans les pays dével oppés. Permanences et changements, p. 259-277. París: France Prioux (ed.), IN ED.

INE (1978). Encuesta de Fecundidad. M adrid.

- (1987). Encuesta de Fecundidad, 2 vols. M adrid.

J OSHI, H eather (1990). «T he C ash O pportunity C osts of Childbearing: An Approach to Estimation U sing British D ata». Population Studies, vol. 44, p. 41-60.

KraVDAL, O ystein (1994). «T helmportance of Economic Activity, Econmic Potential and Economic Resources for the Timing of First Births in N orway». Population Studies, vol. 48, p. 249-267.

Ley 22 de noviembre de 1988. Reproducción Asistida H umana, Regula las T écnicas. Boletín 0 ficial del Estado, 35/1988, núm. 281-282, p. 5622-5630.

Lo GAN , John R.; SPITZE, G lenna D . (1994). «Family N eighbors». American Journal of Sociology, vol. 100, núm. 2, p. 453-476.

M ASO N, Karen O ppenheim (1995). «G ender and D emographic Change: W hat do we know?». IUSSP, $31 \mathrm{p}$.

M отт, Frank L. (1990). «W hen Is a Father Really Gone? Paternal-C hild Contact in Father-Absent H omes». D emography, vol. 27, núm. 4, p. 499-517.

$O$ 'M ALLEY BORG, M ary (1989). «T he Income-Fertility Relationship: Effet of the $N$ et Price of a C hild». D emography, vol. 26, núm. 2, p. 301-310.

Presser, H arriet B. (1989). «C an We M ake Time for Children? The Economy, Work Schedules and Child Care». D emography, vol. 26, núm. 4, p. 523-543.

Ro usSEL, Louis (1991). «Les futuribles de la famille». Futuribles, p. 3-21.

SALAS, Rafael M . (1985). 